\title{
Hyperpolarized Carbon C 13 Pyruvate
}

National Cancer Institute

\section{Source}

National Cancer Institute. Hyperpolarized Carbon C 13 Pyruvate. NCI Thesaurus. Code C116853.

A hyperpolarized pyruvate labeled with carbon C 13, with potential usage in the diagnostic imaging of cancer cells. Upon administration, carbon C 13 hyperpolarized pyruvate (13Cpyruvate) is taken up by cancer cells and is further metabolized to lactate and alanine for use in glycolysis, lipogenesis and protein synthesis, to support the enhanced metabolic needs of tumor cells. As tumor cells are rapidly proliferating, their uptake of pyruvate and its subsequent conversion to alanine and lactate are higher than non-proliferating cells. Upon nuclear magnetic resonance (NMR)-based imaging (MRI), both hyperpolarized 13Cpyruvate as well as its metabolites can be detected and visualized in cancer cells; this may aid in the diagnosis of cancer. Hyperpolarization of 13C-pyruvate, using dynamic nuclear polarization (DNP), enhances NMR signals. 